\title{
Development and application of a short- /long-term composited drought index in the upper Huaihe River basin, China
}

\author{
M. Yu ${ }^{1,2}$, Q. $\mathbf{L i}^{1,2}$, G. Lu ${ }^{1}, \mathbf{H}$. Wang ${ }^{3}$, and P. $\mathbf{L i}^{4}$ \\ ${ }^{1}$ College of Hydrology and Water Resources, Hohai University, Nanjing 210098, China \\ ${ }^{2}$ Center for International River Research, School of Business, Hohai University, Nanjing 210098, China \\ ${ }^{3}$ Xinyang Hydrology and Water Resources Survey Bureau, Xinyang, China \\ ${ }^{4}$ Henan Hydrology and Water Resources Survey Bureau, Zhengzhou, China
}

Correspondence to: M. Yu (meixiuyu1985@gmail.com)

Received: 15 April 2015 - Accepted: 15 April 2015 - Published: 11 June 2015

\begin{abstract}
Accurate and reliable drought monitoring is of primary importance for drought mitigation and reduction of social-ecological vulnerability. The aim of the paper was to propose a short-term/long-term composited drought index (CDI) which could be widely used for drought monitoring and early warning in China. In the study, the upper Huaihe River basin above the Xixian gauge station, which has been hit by severe droughts frequently in recent decades, was selected as the case study site. The short-term CDI was developed by the Principle Component Analysis of the self-calibrating Palmer Drought Severity Index (sc-PDSI), the 1- and 3-month Standardized Precipitation Evapotranspiration Index (SPEI), Z Index (ZIND), the Soil Moisture Index (SMI) with the long-term CDI being formulated by use of the self-calibrating Palmer Hydrology Drought Index (sc-PHDI), the 6-, 12-, 18- and 24-month SPEI, the Standardized Streamflow Index (SSI), the SMI. The sc-PDSI, the PHDI, the ZIND, the SPEI on a monthly time scale were calculated based on the monthly air temperature and precipitation, and the monthly SMI and SSI were computed based on the simulated soil moisture and runoff by the distributed Xinanjiang model. The thresholds of the short-term/long-term CDI were determined according to frequency statistics of different drought indices. Finally, the feasibility of the two CDIs was investigated against the scPDSI, the SPEI and the historical drought records. The results revealed that the short-term/long-term CDI could capture the onset, severity, persistence of drought events very well with the former being better at identifying the dynamic evolution of drought condition while the latter better at judging the changing trend of drought over a long time period.
\end{abstract}

\section{Introduction}

Drought is one of the most damaging natural hazards and often results in devastating effects to social and ecological systems (Hao and Aghakouchak, 2014). The annual economic damage of severe droughts across China is estimated to be $2.5-3.5 \%$ of the gross domestic product. The frequency of severe and extreme droughts in China increased significantly from $26.8 \%$ during $1950-1990$ to $47.6 \%$ during 1991-2011, and the drought percentage area increased by $3.92 \% / 10 \mathrm{a}$ from 1990s (Yu et al., 2014). Thus, accurate and reliable drought monitoring and prediction is of critical im- portance for risk assessment and decision making. Different drought indices have been developed and applied for drought monitoring and assessment. The Palmer drought severity index (PDSI; Palmer, 1965), self-calibrating PDSI (Wells et al., 2004) are widely used for drought characterization. The standardized precipitation index (SPI; McKee et al., 1993) and the standardized precipitation evapotranspiration index (SPEI; Vicente-Serrano et al., 2010) are commonly used for meteorological drought monitoring. The standardization concept was also applied to other drought indices such as the standardized soil moisture index (SMI) and the standardized streamflow index (SSI, Vicente-Serrano et al., 2012). How- 
ever, the performance of different drought index differs in detecting the drought onset, persistence and termination. Wilhite (2005) concluded that using of a single index to reveal the diversity and complexity of drought conditions and impact is one of the major limitations to drought monitoring. Due to the fact that no single index can represent all aspects of meteorological, agricultural and hydrological droughts, a multi-index approach should be proposed for drought monitoring, assessment and prediction (Hao and Aghakouchak, 2014). The aim of this paper is to introduce and evaluate a short-term/long-term composited drought index (short-term CDI/ long-term CDI) in the upper Huaihe River basin. Located in a transition zone between the climates of North and South China, the Huaihe River basin was determined to be the region being easily hit by floods and droughts frequently. The output of the study could provide valuable references for the drought monitoring and early warning system development in the Huaihe River basin and other regions/basins in China.

\section{Short- /long-term CDI development}

\subsection{Selection of CDI components}

Based on the differences in time scales and drought types of drought index, the self-calibrating Palmer Drought Severity Index (scPDSI), the 1- and 3-month Standardized Precipitation Evapotranspiration Index (SPEI), Z Index (ZIND), the Soil Moisture Index (SMI) were selected to develop the short-term CDI by using the Principle Component Analysis (PCA), while the self-calibrating Palmer Hydrology Drought Index (scPHDI), the 6-, 12-, 18- and 24-month SPEI, the Standardized Streamflow Index (SSI), the SMI were chosen to formulate the long-term CDI. The dynamic evolution of drought condition can be obtained by the short-term CDI while the changing trend of drought over a long time period could be known by the long-term CDI.

\subsection{Principle component analysis}

The principal component analysis (PCA) method is a technique applied to multivariate analysis for dimensionality reduction. The original intercorrelated variables could be reduced to a small number of new linearly uncorrelated ones that explain most of the total variance.

Considering $k$ variables in a given time period $i, X i, 1$, $X i, 2, \ldots, X i, k, k$ principle components (PCs) are produced for the same time period, $Y_{i, 1}, Y_{i, 2}, \ldots, Y_{i, k}$, using linear combinations of the first ones, according to:

$\left\{\begin{array}{c}Y_{i, 1}=e_{11} X_{i, 1}+e_{12} X_{i, 2}+\ldots+e_{1 k} X_{i, k} \\ Y_{i, 2}=e_{21} X_{i, 1}+e_{22} X_{i, 2}+\ldots+e_{2 k} X_{i, k} \\ \quad \ldots \ldots \\ Y_{i, k}=e_{k 1} X_{i, 1}+e_{k 2} X_{i, 2}+\ldots+e_{k k} X_{i, k}\end{array}\right.$
In the applications the variables $X_{i, k}$ refer to scPDSI, SPEI1, SPEI3, ZIND, SMI, series for short-term CDI, and refer to scPHDI, SPEI6 ,SPEI12, SPEI18, SPEI24, SSI, SMI series for long-term CDI. $k$ is equal to the number of drought indices ( 5 for short-term CDI and 7 for long-term CDI) and $i$ represents the length of drought indices series.

In Eq. (1) the $Y_{i, 1}$ explains most of the variance, $Y_{i, 2}$ explains the reminiscent amount of variance, and so on. PCs extraction could be based on variance/covariance or correlation matrix of data with $\left\{e_{11}, e_{21}, \ldots, e_{1 k}\right\}$ being the first eigenvector and $\left\{e_{11}, e_{21}, \ldots, e_{1 k}\right\}$ being the eigenvector of $k$ order. Each eigenvector includes the coefficients of the $k$ principal component. Finally, the amount of variance explained by the first PC is called the first eigenvalue $\lambda_{1}$, the second is $\lambda_{2}$, so that $\lambda_{1} \geq \lambda_{2} \ldots \geq \lambda_{k}$. Since each eigenvalue represents the fraction of the total variance in the original data and explained by each component, proportion of each component can be calculated as $\lambda_{j} / \sum \lambda_{j}$. Thus, the short-term/long term CDI can be expressed as follows:

$$
\begin{aligned}
\mathrm{CDI}_{i} & =\frac{\lambda_{1}}{\lambda_{1}+\lambda_{2}+\ldots+\lambda_{k}} Y_{i, 1}+\ldots \\
& +\frac{\lambda_{j}}{\lambda_{1}+\lambda_{2}+\ldots+\lambda_{k}} Y_{i, j}+\ldots \\
& +\frac{\lambda_{n}}{\lambda_{1}+\lambda_{2}+\ldots+\lambda_{k}} Y_{i, k},
\end{aligned}
$$

where $\lambda_{j}$ is eigenvalue of the $j$ th principal component, $Y_{i, j}$ is the $j$ th principal component of the $i$ th month, $\mathrm{CDI}_{i}$ is the short-term/long-term CDI in the $i$ th month.

\subsection{Short-/long-term CDI formulation}

According to Eqs. (1) and (2), the short-term CDI can be formulated as follows:

$$
\begin{aligned}
& \mathrm{S}_{-} \mathrm{CDI}_{i}=\sum_{1}^{k} \frac{\lambda_{j}}{\lambda_{1}+\lambda_{2}+\ldots+\lambda_{k}} X_{\mathrm{S}} E_{\mathrm{S}} \\
& X_{\mathrm{S}}=\left(x_{\mathrm{ScPDSI}, i}, x_{\mathrm{ZIND}, i}, x_{\mathrm{SPEI} 1, i}, x_{\mathrm{SPEI} 3, i}, x_{\mathrm{SMI}, i}\right) ; \\
& E_{\mathrm{S}}=\left(e_{\mathrm{ScPDS}, i, j}, e_{\mathrm{ZIND}, i, j}, e_{\mathrm{SPEI} 1, i, j}, e_{\mathrm{SPEI}, i, j}, e_{\mathrm{SMI}, i, j}\right)^{T}
\end{aligned}
$$

And the long term CDI can be formulated as follows:

$$
\begin{aligned}
\mathrm{L}_{-} \mathrm{CDI}_{i}= & \sum_{1}^{k} \frac{\lambda_{j}}{\lambda_{1}+\lambda_{2}+\ldots+\lambda_{k}} X_{\mathrm{L}} E_{\mathrm{L}} \\
X_{\mathrm{L}}= & \left(x_{\mathrm{SMI}, i}, x_{\mathrm{SCPHDI}, i}, x_{\mathrm{SSI}, i}, x_{\mathrm{SPEI} 6, i}, x_{\mathrm{SPEI} 12, i},\right. \\
& \left.x_{\mathrm{SPEI} 18, i}, x_{\mathrm{SPEI} 24, i}\right) ; \\
E_{\mathrm{L}}= & \left(e_{\mathrm{SMI}, i, j}, e_{\mathrm{PHDI}, i, j}, e_{\mathrm{SSI}, i, j}, e_{\mathrm{SPEI} 6, i, j},\right. \\
& \left.e_{\mathrm{SPEI} 12, i, j}, e_{\mathrm{SPEI} 18, i, j}, e_{\mathrm{SPEI} 24, i, j}\right)^{T},
\end{aligned}
$$

where $\lambda_{j}$ are eigenvalues of the $j$ th principal component, $e_{i, j}$ are eigenvectors of the $j$ th principal component of the $i$ th month, $X_{i}$ is the drought index series in the $i$ th month, 


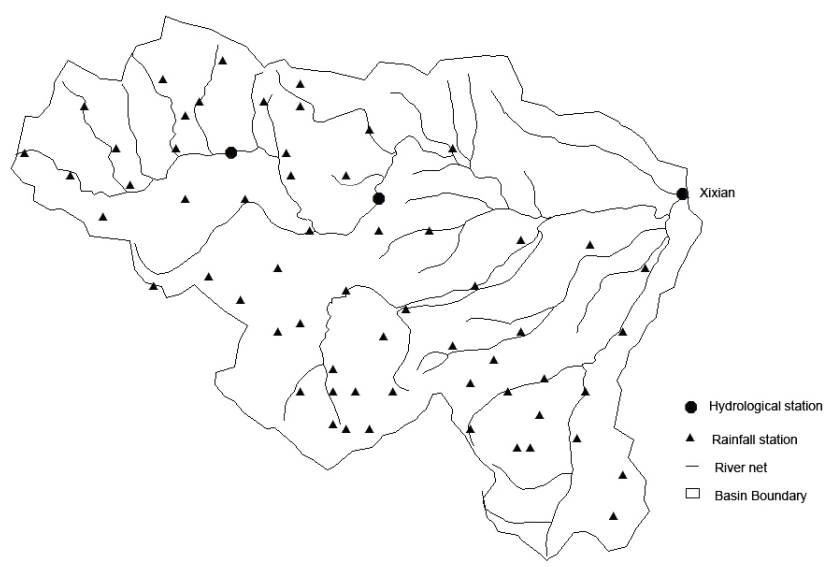

Figure 1. Sketch map of the upper Huaihe River basin.

S_CDI $i$ and L_CDI $i$ are the short-term CDI and the longterm CDI in the $i$ th month.

It should be noted that the monthly scPDSI, ZIND, scPHDI and SPEI (in time scales of 1-month, 3-month, 6month, 12-month, 18-month, 24-month respectively) could be obtained based on the daily air temperature and precipitation from meteorological stations in/around the study basin, while the monthly SMI and the SSI need to be calculated by soil water volume and generated runoff based on the hydrological modelling. The gridded Xinanjiang model (XAJ; Zhao, 1992) - model of runoff formation on saturation of storage, used widely in humid and semi-humid regions in China, was adopted to simulate the gridded soil water and generated runoff in the upper Huaihe River basin.

\subsection{Determination of the drought intensity threshold levels of the CDI categories}

The drought intensity threshold levels of the short-term CDI was conducted as following steps: firstly, calculate the drought event frequency $F_{i, j}$ ( $i$ for the drought intensity level, $j$ for different drought index) of all the short-term drought components with different drought intensity level in the upper Huaihe River basin above the Xixian station; secondly, average the $F_{i, j}$ for the same drought intensity level, then a series of new drought frequency $f_{i}$ ( $i$ for the drought intensity level) of different drought intensity levels for the short-term CDI was obtained; thirdly, determine the drought intensity threshold levels of the short-term CDI according to the $f_{i}$. The drought intensity threshold levels of the long-term CDI can also be acquired by following the above steps.

\section{Application of the short- /long-term CDI}

\subsection{Case study site}

The Huaihe River Basin is one of seven major river basins in China, and lies in the warm temperature semi-humid mon-

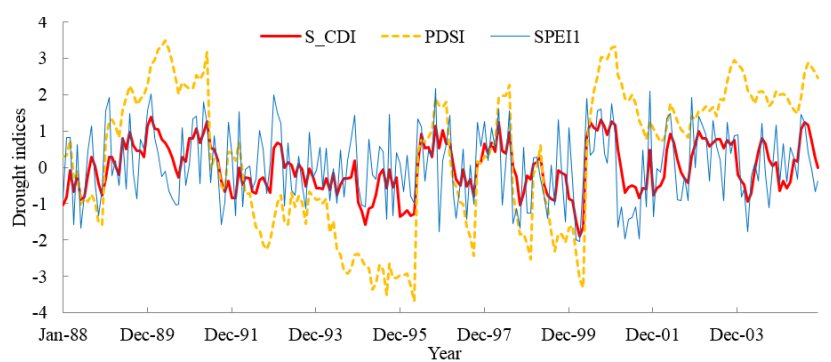

Figure 2. Temporal variations of the short-term CDI and its components.

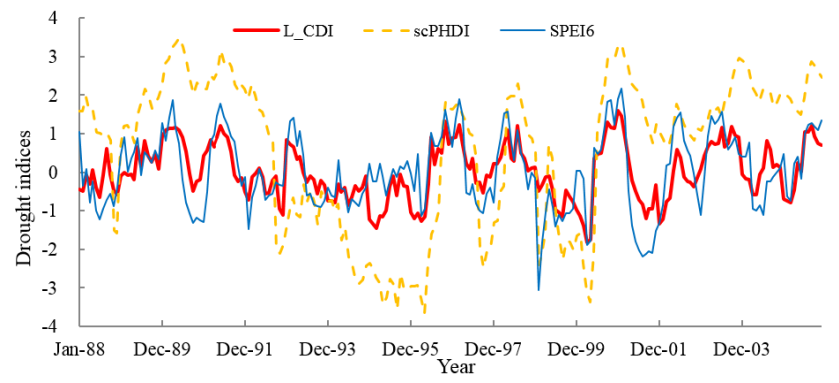

Figure 3. Temporal variations of the long-term CDI and its components.

soon region, which is a transition zone between the climates of North and South China. Special geographical location determined that the Huaihe River basin was a region easily hit by floods and droughts over many centuries, particularly in recent decades, severe droughts occurred much more frequently which caused huge economic and social loss. To propose a short- /long-term CDI which could be widely used for drought monitoring and early warning in China, this paper selected the upper Huaihe River basin above Xixian gauge station as the case study site with a catchment area of $10190 \mathrm{~km}^{2}$ (see Fig. 1)

\subsection{Data preparation and processing}

Considering the spatial unevenness of underlying conditions and meteorological variables, the study areas were divided into grids with a size of $0.873 \mathrm{~km} \times 0.873 \mathrm{~km}$. Based on the daily observed meteorological variables (precipitation and air temperature) from closer six meteorological stations during 1988 to 2005, the inverse distance-squared weighted method was used to acquire the meteorological data for every grid. Before the interpolation, digital elevation model was used to correct the meteorological variables according to meteorological variable-elevation relationships. Monthly gridded scPDSI, ZIND, scPHDI and SPEI (1-month, 3-month, 6-month, 12-month, 18-month, 24-month respectively) was calculated on the basis of the gridded meteorological variables, then the monthly areal drought indices were obtained by averaging their gridded values. 


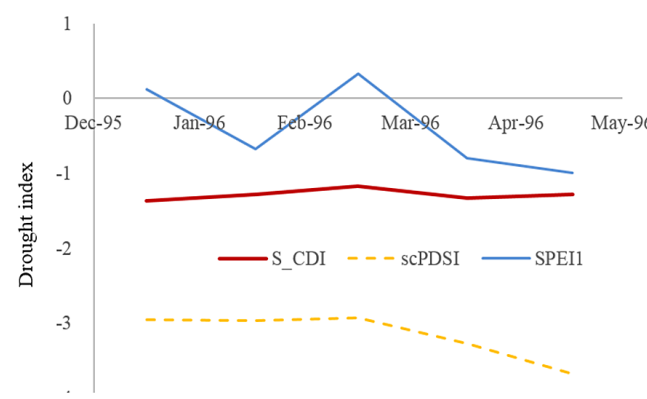

(a) S_CDI

Figure 4. The 1996 drought.

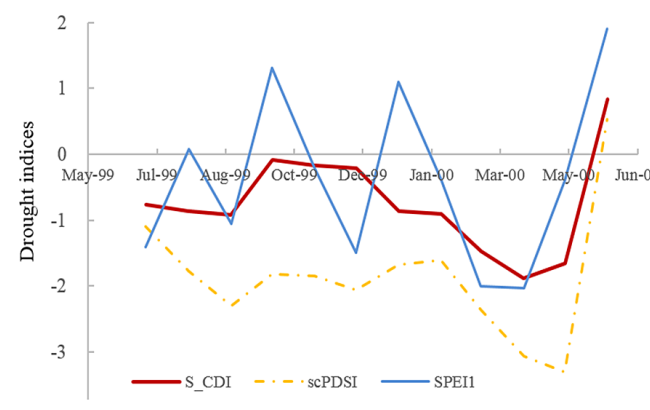

(a) S_CDI

Figure 5. The 2000 drought.

\subsection{Application of the XAJ model}

Based on the topography, hydrological and meteorological data in 1988-2005, the daily gridded XAJ model was adopted to simulate gridded soil water and generated runoff in the upper Huaihe River basin above Xixian station. The calibration and validation periods were 1988-2002 and 2003-2005 respectively (Yu, 2013). The result revealed that the daily XAJ model was simulated with satisfactory accuracy, which indicated that the calculated gridded soil water and generated runoff could be used for the further CDI construction. The monthly gridded SMI and SSI was computed based on the gridded soil water and generated runoff, then the areal monthly SMI and SSI was carried out by averaging the gridded values.

\subsection{Short-/long-term CDI application}

Based on the time series of areal monthly scPDSI, ZIND, 1-mon SPEI, 3-mon SPEI and SMI, according Eq. (3), the short-term CDI in the upper Huaihe River basin above the Xixian station was carried out. The eigenvalues and eigenvectors in Eq. (3) can be referred to Table 1. And the drought intensity threshold levels of the short-term CDI were determined according to Sect. 2.4 (Table 2).

Based on the time series of areal monthly scPHDI, 6mon SPEI, 12-mon SPEI, 18-mon SPEI, 24-mon SPEI, SSI

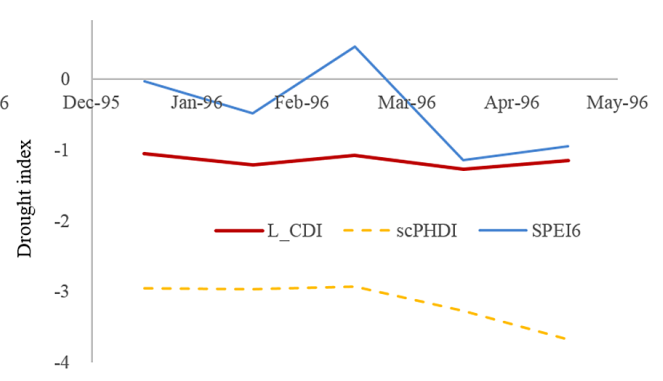

(b)L_CDI

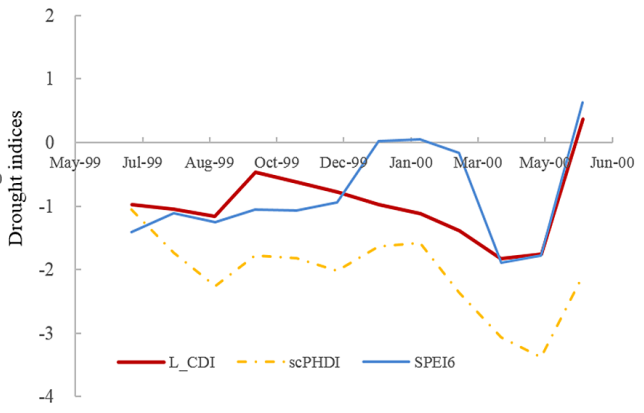

(b)L_CDI

and SMI, according Eq. (4), the long-term CDI in the upper Huaihe River basin above the Xixian station was carried out. The eigenvalues and eigenvectors in Eq. (4) can be referred to Table 1. And the drought intensity threshold levels of the long-term CDI were determined according to Sect. 2.4 (Table 2).

The short-term CDI is suggested to be adopt to the drought assessment during drought initial period while the long-term $\mathrm{CDI}$ is used after the drought persisting for a relatively long time period.

\section{Results and discussion}

\subsection{Temporal variations of the short-term/long-term CDI and other drought index}

The chronological comparison between the S_CDI and the scPDSI, the S_CDI and the 1-mon SPEI time series in the upper Huaihe River basin above Xixian station during 19882005 was displayed in Fig. 2. It can been found that the three drought index identified most dry conditions of the upper Huaihe River basin, the drier the conditions, the lower the drought index values and the basin was in relatively dry condition in 1990s and early 2000s. Generally, the temporal variation of the S_CDI behaved similarly with that of the scPDSI and the 1-mon SPEI, which was also verified by the Spearman correlation between the S_CDI and the scPDSI, and the 
Table 1. Eigenvalues and eigenvectors of the PCA for short-term/long-term CDI in the upper Huaihe River basin.

\begin{tabular}{|c|c|c|c|c|c|c|c|c|c|c|c|}
\hline CDI & $\begin{array}{r}\text { Eig } \\
\lambda_{1} \lambda_{2} \lambda_{3}\end{array}$ & nvalue & & & & & Eige & values $\mathrm{e}_{1}$, & $2, e_{3}$ & & \\
\hline \multirow{3}{*}{ S_CDI } & \multirow{3}{*}{2.82} & \multirow{3}{*}{0.8} & \multirow{3}{*}{0.69} & $e_{1}$ & $\begin{array}{c}e_{1 \mathrm{scPDSI}} \\
0.23\end{array}$ & $\begin{array}{c}e_{1 \mathrm{ZIND}} \\
0.30\end{array}$ & $\begin{array}{c}e_{1 \text { SPEI } 1} \\
0.23\end{array}$ & $\begin{array}{c}e_{1 \text { SPEI }} \\
0.28\end{array}$ & $\begin{array}{c}e_{1 \mathrm{SMI}} \\
0.28\end{array}$ & & \\
\hline & & & & $e_{2}$ & $\begin{array}{c}e_{2 \text { scPDSI }} \\
0.75\end{array}$ & $\begin{array}{c}e_{2 Z \text { ZIND }} \\
-0.21\end{array}$ & $\begin{array}{c}e_{2 S P E I} 1 \\
0.48\end{array}$ & $\begin{array}{c}e_{2 S P E I 3} \\
-0.62\end{array}$ & $\begin{array}{l}e_{2 S M I} \\
-0.16\end{array}$ & & \\
\hline & & & & $e_{3}$ & $\begin{array}{c}e_{3 \mathrm{sc} \_P D S I} \\
-0.65\end{array}$ & $\begin{array}{c}e_{3 Z I N D} \\
-0.42\end{array}$ & $\begin{array}{c}e_{3 \text { SPEI }} \\
0.85\end{array}$ & $\begin{array}{c}e_{3 \text { SPEI3 }} \\
-0.08\end{array}$ & $\begin{array}{c}e_{3 \mathrm{SMI}} \\
0.36\end{array}$ & & \\
\hline \multirow{3}{*}{ L_CDI } & \multirow{3}{*}{3.77} & \multirow{3}{*}{1.24} & \multirow{3}{*}{0.6} & $e_{1}$ & $\begin{array}{c}e_{1 \mathrm{SMI}} \\
0.61\end{array}$ & $\begin{array}{c}e_{1 \mathrm{scPHDI}} \\
0.71\end{array}$ & $\begin{array}{c}e_{1 \mathrm{SSI}} \\
0.75\end{array}$ & $\begin{array}{c}e_{1 \text { SPEI } 6} \\
0.81\end{array}$ & $\begin{array}{c}e_{1 \text { SPEI12 }} \\
0.76\end{array}$ & $\begin{array}{c}e_{1 \text { SPEI1 }} 8 \\
0.72\end{array}$ & $\begin{array}{c}e_{1 \text { SPEI24 }} \\
0.76\end{array}$ \\
\hline & & & & $e_{2}$ & $\begin{array}{c}e_{2 \mathrm{SMI}} \\
0.59\end{array}$ & $\begin{array}{c}e_{2 \mathrm{scPHDI}} \\
-0.28\end{array}$ & $\begin{array}{c}e_{2 S S I} \\
0.32\end{array}$ & $\begin{array}{c}e_{2 \text { SPEI6 }} \\
-0.18\end{array}$ & $\begin{array}{c}e_{2 \text { SPEI12 }} \\
-0.48\end{array}$ & $\begin{array}{c}e_{2 \text { SPEI18 }} \\
-0.41\end{array}$ & $\begin{array}{c}e_{2 \text { SPEI24 }} \\
0.53\end{array}$ \\
\hline & & & & $e_{3}$ & $\begin{array}{c}e_{3 \mathrm{SMI}} \\
0.43\end{array}$ & $\begin{array}{c}e_{3 \mathrm{scPHDI}} \\
0.27\end{array}$ & $\begin{array}{c}e_{3 S S I} \\
-0.51\end{array}$ & $\begin{array}{c}e_{3 \text { SPEI6 }} \\
-0.27\end{array}$ & $\begin{array}{c}e_{3 \text { SPEI12 }} \\
0.09\end{array}$ & $\begin{array}{c}e_{3 \text { SPEI18 }} \\
0.08\end{array}$ & $\begin{array}{c}e_{3 \text { SPEI24 }} \\
0.03\end{array}$ \\
\hline
\end{tabular}

Notes: $\lambda_{1}, \lambda_{2}, \lambda_{3}$ are eigenvalues, $e_{1}, e_{2}, e_{3}$ are eigenvectors.

Table 2. Drought categories of the short-term/long-term CDI.

\begin{tabular}{lrr}
\hline Drought Category & Short-term CDI & Long-term CDI \\
\hline Normal & $(-0.37,+\infty)$ & $(-0.33,+\infty)$ \\
Mild drought & $(-0.69,-0.37]$ & $(-0.78,-0.33]$ \\
Moderate drought & $(-0.97,-0.69]$ & $(-1.16,-0.78]$ \\
Severe drought & $(-1.36,-0.97]$ & $(-1.39,-1.16]$ \\
Extreme drought & $(-\infty,-1.36]$ & $(-\infty,-1.39]$ \\
\hline
\end{tabular}

S_CDI and the 1-mon SPEI time series with their correlation coefficients being of $0.83,0.72$ respectively.

The chronological comparison between the L_CDI and the scPHDI, the L_CDI and the 6-mon SPEI time series was shown in Fig. 3. Generally, the temporal variation of the L_CDI behaved similarly with that of the scPHDI and the 6-mon SPEI, which was also verified by the Spearman correlation between the L_CDI and the scPHDI, and the L_CDI and the 6-mon SPEI time series with their correlation coefficients being of $0.75,0.82$ respectively.

Clearly, the variation trend of all the drought index are consistent with each other, however, the drought assessment details by the drought index differed for some typical drought events.

\subsection{Drought assessment of critical drought events}

Two critical drought events - the long-term 2000 drought and the short-term 1996 drought were used to assess the fidelity of the S_CDI, the L_CDI and their drought components.

\subsubsection{Historical records of critical droughts}

The 2000 drought, which actually began from the September of 1999 and developed to the severe spring and early summer drought in 2000, was one of the most famous extreme droughts in the Huaihe River basin in history. During this drought, the total precipitation during January-May of 2000 was less than $45 \%$ of normal, no flow in the main river during April and May. According to incomplete statistics, the precipitation in Shangdong province - one of the major provinces in the Huaihe River basin decreased 84 percent of normal during March and April, 50\% large and middlesized reservoirs in the Province were operated under dead storage level and all the small reservoirs were depleted. The extreme drought in 1996 was also chosen to assess the feasibility of the newly developed CDI. Fortunately this drought event only persisted for a short period.

\subsubsection{Drought in 1996}

From Fig. 4a, it can be found that severe and extreme droughts in early 1996 were identified by the S_CDI, while only moderate and severe droughts were identified by the scPDSI and no drought by the 1-mon SPEI. From Fig. 4b, only moderate and severe droughts were recognized by the L_CDI and the scPHDI, and mild drought by the 6-mon SPEI. Compared with the historical records, it can be concluded that the S_CDI best described the actual dry condition of the short-term drought event in 1996. It indicated that the S_CDI could well capture the relative timely dynamic variation of the short-term drought evolution.

\subsubsection{Drought in 2000}

Both the S_CDI and the 1-mon SPEI identified extreme droughts in March and April of 2000. However, the extreme drought continued developing till May of 2000 and it was only detected by the S_CDI, while no drought was found by the 1-mon SPEI (Fig. 5a). In the meanwhile, severe drought was detected by the scPDSI. From Fig. 5b, the L_CDI iden- 
tified extreme droughts in March-May of 2000 while the 6mon SPEI identified this extreme drought only in April and May. Moreover, moderate drought was also detected from November of 1999 to January of 2000 by the L_CDI, while almost no drought occurred identified by the 6-mon SPEI. Severe drought was detected by the scPHDI only in April and May. The above comparison demonstrated that the S_CDI and L_CDI best characterized the extreme drought in 2000. Since this drought persisted a relatively long time period, the L_CDI was better identifying the whole drought evolution since the L_CDI detected the severe drought in September of 1999 while the S_CDI did not judge accurately.

\section{Conclusions}

The short-term/long-term CDI were developed and applied in the upper Huaihe River basin. The performance of the shortterm/long-term CDI were compared with their drought index components respectively. The result revealed that the newlybuilt short-term CDI could better capture the drought severity, onset, and termination than the scPDSI and the 1-mon SPEI for relatively short-term droughts, while the long-term CDI behaved better than the scPHDI and the 6-mon SPEI on drought persistence, magnitude for relatively long-term droughts.

Acknowledgements. Financial support is gratefully acknowledged from the National Science Foundation Commission under Grant No. 41171220, China, the project (2014B04314) supported by the Fundamental Research Funds for the Central Universities.

\section{References}

Hao, Z. C. and Aghakouchak, A.: A nonparametric multivariate multi-index drought monitoring framework, J. Hydrometeorol., 15, 89-101, 2014.

McKee, T., Doesken, N., and Kleist, J.: The relationship of drought frequency and duration to time scales. Preprints, Eight Conf. on Applied Climatology. Anaheim, CA, Am. Meteorol. Soc., 179184, 1993.

Palmer, W.: Meterological droughts, US Department of Commerce, Weather Bureau Research Paper 45, 58, 1965.

Vicente-Serrano, S. M., Beguería, S., and López-Moreno, J.: A multiscalar drought index sensitive to global warming: The Standardized Precipitation Evapotranspiration Index, J. Climate, 23, 1696-1718, 2010

Vicente-Serrano, S. M., López-Moreno, J. I., and Beguería, S.: Accurate Computation of a Streamflow Drought Index, J. Hydrol., 17, 318-332, 2012.

Wells, N., Goddard, S., and Hayes, M. J.: A Self-Calibrating Palmer Drought Severity Index, J. Climate, 17, 2335-2351, 2004.

Wilhite, D. A.: Drought and Water Crises: Science, Technology, and Management Issues, Taylor and Francis, 406 pp., 2005.

Yu, M. X.: Development and application of the Composited Drought Index, PhD, Hohai University, Nanjing, China, 2013.

Yu, M. X., Li, Q. F., Hayes, M. J., Svoboda, M. D., and Heim, R. R.: Are droughts becoming more frequent in China based on the SPEI: 1951-2010?, Int. J. Climatol., 34, 545-558, 2014.

Zhao, R. J.: The Xinanjiang model applied in China, J. Hydrol., 135, 371-381, 1992. 